\title{
Sudden deafness as an initial presentation of varicella: case report and literature review
}

\author{
Minmin Shao, Guofeng Xiong, Guangzao Xiang, Shile Xu, Yingsong Zheng, Liqun Zhang \\ Department of Otorhinolaryngology, The Second Affiliated Hospital of Shanghai University (Wenzhou Central Hospital), Wenzhou, China \\ Correspondence to: Liqun Zhang. Department of Otorhinolaryngology, The Second Affiliated Hospital of Shanghai University, Wenzhou 325000, \\ China. Email: weyzlq@qq.com.
}

\begin{abstract}
Varicella, or chickenpox, is a highly contagious disease transmitted by the varicella zoster virus (VZV). Idiopathic sudden sensorineural hearing loss (ISSNHL) has been defined as a loss of at least $30 \mathrm{~dB}$ over at least three frequencies on audiogram within three days and with an uncertain cause. Deafness caused by varicella-zoster virus occasionally occurred in Ramsey hunter syndrome but associated with chickenpox is rare. We present a case of an adult varicella infection with sudden deafness as the first symptom. The patient begins with a sudden hearing loss in his left ear and developed fever 12 days later and skin rash 13 days later. He was diagnosed as chickenpox and received treatment of oral famciclovir capsule $250 \mathrm{mg}$ administrated three times daily. After 2 weeks, at an outpatient follow-up the patient reported that while his scabs had fallen off and he felt well, the sudden deafness in the left ear had not improved. This kind of cases are rare and suggest the relationship between VZV and sudden deafness. Sudden deafness can be the first symptom of chickenpox. In this study, we also reviewed pathogenesis, clinical feature and the epidemiological character of chickenpox, the neurological complications of varicella zoster and the etiology of sudden deafness.
\end{abstract}

Keywords: Sudden deafness; varicella; varicella-zoster virus; case report

Submitted Mar 10, 2021. Accepted for publication May 06, 2021.

doi: 10.21037/apm-21-785

View this article at: http://dx.doi.org/10.21037/apm-21-785

\section{Introduction}

Varicella or chickenpox is an acute infection caused by the varicella zoster virus (VZV). VZV is double-stranded DNA virus which belong to the alphaherpes sub-family of viruses which can both infect humans and establish longterm dormancy in the dorsal root ganglion (1). The main transmission route of primary varicella infection is contact with respiratory secretions or the vesicle fluid of active varicella patients (2) and while the incubation period from infection to the onset of clinical symptoms is generally 10 to 21 days, patients are infectious and can transmit the virus through droplets 1 or 2 days before the initial skin eruption (3).

Varicella mainly occurs in children under the age of 10 years in whom it presents as a blister pruritus rash which begins on the trunk and gradually extends to the head, face, and limbs. There are well-formed, contagious viruses in skin vesicles, which can form aerosols and transmit VZV to others who have not been previously infected with VZV. Concomitant symptoms include malaise, fever, fatigue, and loss of appetite, and the illness usually lasts for one to two weeks although complications including secondary bacterial infection, pneumonia, encephalitis, hepatitis, and Raynaud's syndrome can occur. Adults and patients with underlying diseases are more likely to have more serious complications than children $(4,5)$, including several neurological complications (6-8). We report a case of a previously healthy immunocompetent 38 -year-old man with sudden deafness as the initial symptom of varicella. This kind of cases are rare and suggest the relationship between viral infection and sudden deafness.

We present the following article in accordance with the CARE reporting checklist (available at http://dx.doi. org/10.21037/apm-21-785). 


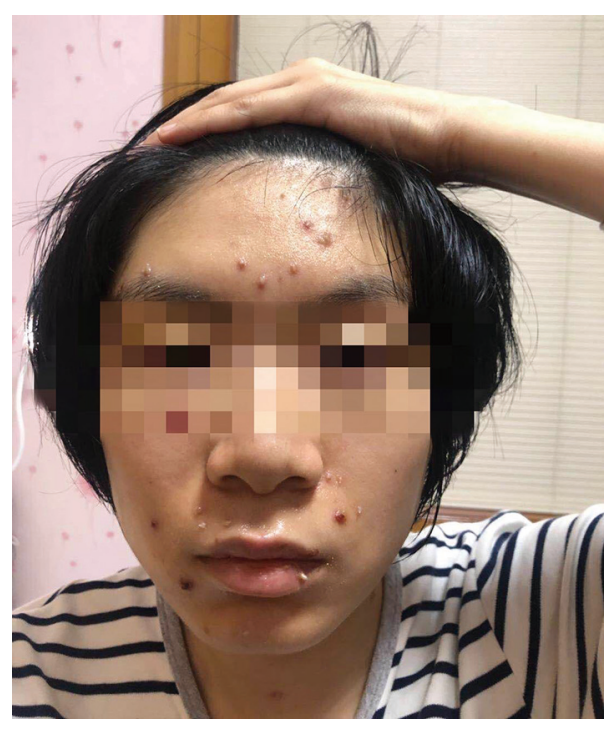

Figure 1 Photograph of the patient taken 1 week after rash onset.

\section{Case presentation}

All procedures performed in studies involving human participants were in accordance with the ethical standards of the institutional and/or national research committee(s) and with the Helsinki Declaration (as revised in 2013). The study was approved by the Ethics Committee of The Second Affiliated Hospital of Shanghai University. Written informed consent was obtained from the patient.

A previously healthy immunocompetent 38-year-old male complained of a 2-day history of left sided hearing loss, continuity low-key tinnitus, and mild vertigo. While on physical examination, otoscopic examination was normal, a pure-tone audiogram showed a hearing threshold of $115 \mathrm{~dB}$ in the left ear and $20 \mathrm{~dB}$ in the right ear, both in air and bone conducted pure-tone stimuli. Routine blood examination revealed a white cell count of $16.2 \times 10^{9} / \mathrm{L}$, neutrophil ratio $81.0 \%$, lymphocyte ratio $11.8 \%$, monocyte count $1.15 \times 10^{9} / \mathrm{L}$, and monocyte ratio $7.1 \%$, and an inner ear magnetic resonance imaging (MRI) report excluded middle ear and inner ear disease. A diagnosis of left ear sudden sensorineural hearing loss was made, and medical treatment consisted of the intravenous administration of high dose steroids (intravenous drip of methylprednisolone sodium succinate $40 \mathrm{mg}$ ) and peripheral vasodilators in combination with the intravenous administration of dextran and Batroxobin $5 \mathrm{BU}$ intravenous infusion (10 BU for the first time) every other day, for a total of three infusions.

Twelve days after admission the patient developed a fever up to $38.6^{\circ} \mathrm{C}$ and routine blood examination showed a white cell count of $9.1 \times 10^{9} / \mathrm{L}$, neutrophil ratio $78.6 \%$, lymphocyte ratio $11.5 \%$, monocyte count $0.84 \times 10^{9} / \mathrm{L}$, monocyte ratio $9.3 \%$, and CRP $15.1 \mathrm{mg} / \mathrm{L}$. Thirteen days after admission, the patient developed a vesicular skin rash over the trunk that rapidly spread to the limbs, head, and face (Figure 1). The patient was diagnosed as chickenpox and then isolated at home and received antiviral treatment of oral famciclovir capsule $250 \mathrm{mg}$ administrated three times daily. After 2 weeks, at an outpatient follow-up the patient reported that while his scabs had fallen off and he felt well, the sudden deafness in the left ear had not improved.

\section{Discussion}

\section{Chickenpox}

Primary VZV infection begins with the virus replication in epithelial cell in the upper respiratory mucosa. According to the model of VZV cell-associated viremia, VZV enters the upper respiratory tract, replicates in the epithelial cells and infects tonsil $\mathrm{T}$ cells, causing subclinical cell-associated viremia about 4-6 days after infection. During viremia, the virus spreads to the reticuloendothelial tissue for further reproduction and is eventually transported to the skin and mucosa. T cells play an important role in the process of virus replication and transport (9).

Varicella mainly occurs in children under the age of 10 years. Prodromal symptoms including malaise, fever, fatigue, headache, and loss of appetite may appear first and skin rash usually appear in waves 14-21 days after infection. Blister pruritus rash begins on the trunk and gradually extends to the limbs, neck, head, and face. It can also involve mucous membranes of oral, tonsil, gastrointestinal tract, conjunctiva and vagina. The lesions turned into pustules after 12 to 72 hours, which often break down and form scabs. Pustules usually leave no sequelae after the scars falling off, but if scratched, they can infected by Staphylococcus or Streptococcus, and finally leaving permanent scars. The main transmission route of primary varicella infection is contact with respiratory secretions or the vesicle fluid of active varicella patients. Infectivity is strongest 1 to 2 days before eruption and gradually decreases after scab. Adults and patients with underlying diseases especially compromised immunity, congenital immunodeficiency, transplantation, various treatments for malignant or autoimmune disease are more likely to have more serious complications than children without underlying disease $(4,5)$. The most common complications include bacterial superinfection of skin (5\%), encephalitis, 
Table 1 Varicella zoster virus (VZV) nervous system complications

\begin{tabular}{|c|c|}
\hline Neurologic complications & Affected nerve \\
\hline Acute cerebellar ataxia & Uncertain \\
\hline Guillain-Barre' syndrome & Polyradiculopathy \\
\hline Reye syndrome & Uncertain \\
\hline Optic neuritis & Optic nerve \\
\hline Meningoencephalitis & Vasculopathy \\
\hline Vasculitis & $\begin{array}{l}\text { Large vessel unifocal granulomatous arteritis and small vessel } \\
\text { multifocal vasculopathy }\end{array}$ \\
\hline arm weakness & Brachial plexus \\
\hline diaphragmatic paresis & phrenic nerve \\
\hline Neurogenic bladder and loss of anal sphincter control & Lumbosacral Plexus \\
\hline Autonomic dysfunction & Visceral disease and chronic intestinal pseudo-obstruction \\
\hline Postherpetic neuralgia & Any level of the neuraxis can be affected \\
\hline
\end{tabular}

pneumonia, cerebellar ataxia, and other unusual complications such as hepatitis, nephritis, myocarditis, thrombocytopenia (2).

Humans are the only reservoir of Varicella-Zoster virus, and transmission rate is as high as $90 \%$ after exposure to VZV. The most effective method of prevention is vaccination. Varicella Vaccine (VV) is a live-attenuated viral vaccine which was first developed in Japan in the early 1970s (Oka strain). There are several licensed formulations of live attenuated vaccines available now. Long-term protection from the vaccination has been evidenced, the persistence of antibodies can be up to ten years and efficacy is higher than $90 \%(10)$.

\section{VZV complications of the nervous system}

A literature review revealed that as a neurotropic virus, VZV can cause complications of the nervous system (Table 1) $(6,8)$. Autopsy studies of VZV in sensory ganglia dissected from human tissues have found that the VZV genome (approximately 2-9 copies per cell) is present in about $4 \%$ of neurons during latency. When VZV replicates in skin and dorsal root ganglia (DRG) xenografts, VZV genomic
DNA, viral proteins and virion production are detectable in both neurons and satellite cells. VZV can also induce fusion and polykaryocytes formation between differentiated neurons and surrounding satellite cells which amplifies the spread of VZV to neuronal cell bodies in the ganglion. Two glycoproteins of VZV [glycoprotein E (ge) and glycoprotein $\mathrm{I}(\mathrm{gI})]$ have functions that influence VZV pathogenesis in DRG xenografts. Study found that VZV can replicate in DRG in the absence of $\mathrm{gI}$ suggests that the requirements for VZV infection of neural cells are less stringent than those that are required for infection of $\mathrm{T}$ cells or the skin (11). After primary infection, proliferative retrograde VZV transport along the axons of the nervous system to the neuron occurs, which can cause latent infection in the neurons of the dorsal root or cranial nerve ganglia for years even for decades. The virus maintains replication ability during this latency period and when the host's immunity decreases or is affected by other factors, VZV begins centrifugal transport via axons of the nervous system, reactivates, and causes recurrent disease (12).

The most common neurological complication after VZV infection is herpes zoster, which mainly occurs in patients over 60 years or those with compromised immune function 
and from which some patients develop chronic neuralgia sequelae. The Ramsay-Hunt syndrome is associated with deafness and is caused by reactivated VZV which was previously latent in the sensory ganglion of the spinal and cranial nerves. While the typical clinical manifestations are herpes zoster oticus and lower motor neuron facial palsy, because of the complex neural interactions involved in the region, the trigeminal, facial, glossopharyngeal, and vagus nerves can also be affected presenting as a range of symptoms including hyperacusis, nausea, vomiting, vertigo, and difficulty swallowing. While damage to the cochlea is also perceived to cause deafness (13), sudden deafness as initial presentation of the varicella is rare.

\section{Etiology of sudden deafness}

Idiopathic sudden sensorineural hearing loss (ISSNHL) has been defined as a loss of at least $30 \mathrm{~dB}$ over at least three frequencies on audiogram within three days and with an uncertain cause (14). Most cases are unilateral, with bilateral simultaneous or successive involvement accounting for only $0.4-4.9 \%$ of all ISSHL patients (15), and the presenting symptom is often a feeling of fullness or blocked ear, accompanied by tinnitus and dizziness $(16,17)$. The incidence in adults varies from 2 to 30 per 100,000 although in Germany, an incidence of as high as 160 per 100,000 individuals per year has been reported $(18,19)$. While the pathogenesis of ISSNHL is not clear, microcirculation disorder, autoimmune disease, cochlear membrane injury, and viral infection (20) have been implicated in its onset.

Through examining the temporal bone pathology of patients with ISSNHL, studies have found that the pathological changes of specimens were similar to those of known viral cochlear inflammation (21). There are three potential mechanisms that have been proposed to explain how a viral infection can lead to ISSHL. (I) The virus directly infects the cochlea or cochlea nerve by a hematogenous route, cerebrospinal fluid, or via soft tissue such as the middle ear. (II) The latent virus in the inner ear is reactivated. (III) The virus causes sudden deafness by the cross-reaction of antibodies induced by the virus, or triggers the circulatory ligand, leading to the activation of the stress pathway, although the inner ear is not infected by the virus (22).

Serological studies have found that mumps IgM antibody is present in the serum of some ISSNHL patients, which supports the hypothesis that mumps virus causes the disease (23). A viral labyrinthitis mouse model which is established by inoculation of HSV-1 or HSV-2 into the middle ear, found that HSV induced sudden deafness and vestibular neuritis and HSV antigen was detected in the vascular lamina of mice. This study showed that HSV infection may leading to sudden deafness by destroying the Corti organ and its supporting structures (24).

Virus reactivation can also lead to sudden deafness, with Ramsey hunter syndrome being the classic example. A 33-year-old male presented with acute facial paralysis following the onset of herpes vesicular disease in the external auditory canal and then sudden deafness occurs two weeks later. Serological tests showed elevated levels of anti-HSV IgG and IgM (25). Another case is a 61-year-old female with oral herpes disease developed bilateral hearing loss. In the acute phase, the titer of IgM antibody, the primary immune response antibody, was not significantly increased, while the titer of IgG antibody, the re-immune response antibody, significantly increased. These serological markers indicate that the patient has experienced reactivation of previously latent HSV-1 infection (26). Virus infection was considered the most likely etiology of sudden deafness in two case.

Viral infection can also activate the innate immune system of the cochlea, leading to immune-mediated hearing loss. A mouse models infected by cytomegalovirus found that even though virus was completely cleared up, cochlear hair cells still keep decreased, suggesting virus-induced ISSNHL was the result of an immune response (27).

Evidence supporting sudden deafness caused by VZV is as follow: (I) many studies had shown that viral infections including those caused by the Herpesviridae family were possible causes of ISSNHL; (II) varicella-zoster viruses, including VZV and human herpesvirus, are human neurotropic alphaherpes viruses. Ramsay Hunt syndrome occurs by reactivation of the varicella-zoster virus in the geniculate or facial nerves. Due to anatomical associations with other cranial or cervical spinal nerves, various clinical features can present, such as tinnitus, hearing loss, nausea and vomiting, vertigo, and nystagmus (11). (III) Previous studies have found cases of sudden deafness caused by chickenpox. One case occurred in a 14-month-old child who developed sudden deafness after the diagnosis of chickenpox (28). Therefore, it was speculated that sudden deafness was the prodromal first symptom after infection with VZV, although antibody testing which may have confirmed the presence of the virus was unfortunately not performed.

ISSNHL is a clinically common acute symptom in otolaryngology. Although the incidence of ISSNHL has increased around the world in recent years, the etiology of 
the disease is still unclear. This case suggests that varicellazoster virus is the possible pathogenic virus of sudden deafness.

\section{Acknowledgments}

Funding: None.

\section{Footnote}

Reporting Checklist: The authors have completed the CARE reporting checklist. Available at http://dx.doi.org/10.21037/ apm-21-785

Conflicts of Interest: All authors have completed the ICMJE uniform disclosure form (available at http://dx.doi. org/10.21037/apm-21-785). The authors have no conflicts of interest to declare.

Ethical Statement: The authors are accountable for all aspects of the work in ensuring that questions related to the accuracy or integrity of any part of the work are appropriately investigated and resolved. The study was approved by the Ethics Committee of The Second Affiliated Hospital of Shanghai University. All procedures performed in studies involving human participants were in accordance with the ethical standards of the institutional and/or national research committee(s) and with the Helsinki Declaration (as revised in 2013). Written informed consent was obtained from the patient.

Open Access Statement: This is an Open Access article distributed in accordance with the Creative Commons Attribution-NonCommercial-NoDerivs 4.0 International License (CC BY-NC-ND 4.0), which permits the noncommercial replication and distribution of the article with the strict proviso that no changes or edits are made and the original work is properly cited (including links to both the formal publication through the relevant DOI and the license). See: https://creativecommons.org/licenses/by-nc-nd/4.0/.

\section{References}

1. Newman AM, Jhaveri R. Myths and Misconceptions: Varicella-Zoster Virus Exposure, Infection Risks, Complications, and Treatments. Clin Ther 2019;41:1816-22.

2. Freer G, Pistello M. Varicella-zoster virus infection: natural history, clinical manifestations, immunity and current and future vaccination strategies. New Microbiol 2018;41:95-105.

3. Gould JM. Principles and Practice of Pediatric Infectious Diseases. Infection Following Burns 2018:527-532.e2.

4. Kennedy PGE, Gershon AA. Clinical Features of Varicella-Zoster Virus Infection. Viruses 2018;10:609.

5. Gershon AA, Breuer J, Cohen JI, et al. Varicella zoster virus infection. Nat Rev Dis Primers 2015;1:15016.

6. Steiner I, Benninger F. Manifestations of Herpes Virus Infections in the Nervous System. Neurol Clin 2018;36:725-38.

7. Lee SC, $\mathrm{Ng} \mathrm{M}$, Tan CL, et al. Vision loss in an immunocompetent child post varicella infection: A case report. Malays Fam Physician 2020;15:54-7.

8. Nagel MA, Niemeyer CS, Bubak AN. Central nervous system infections produced by varicella zoster virus. Curr Opin Infect Dis 2020;33:273-8.

9. $\mathrm{Ku} \mathrm{CC}$, Zerboni $\mathrm{L}$, Ito $\mathrm{H}$, et al. Varicella-zoster virus transfer to skin by $\mathrm{T}$ Cells and modulation of viral replication by epidermal cell interferon-alpha. J Exp Med 2004;200:917-925.

10. Lo Presti C, Curti C, Montana M, et al. Chickenpox: An update. Med Mal Infect 2019;49:1-8.

11. Zerboni L, Sen N, Oliver SL, et al. Molecular mechanisms of varicella zoster virus pathogenesis. Nat Rev Microbiol 2014;12:197-210.

12. Sauerbrei A. Diagnosis, antiviral therapy, and prophylaxis of varicella-zoster virus infections. Eur J Clin Microbiol Infect Dis 2016;35:723-34.

13. Jeon Y, Lee H. Ramsay Hunt syndrome. J Dent Anesth Pain Med 2018;18:333-7.

14. Chandrasekhar SS, Tsai Do BS, Schwartz SR, et al. Clinical Practice Guideline: Sudden Hearing Loss (Update). Otolaryngol Head Neck Surg 2019;161:S1-45.

15. Bing D, Wang DY, Lan L, et al. Comparison between Bilateral and Unilateral Sudden Sensorineural Hearing Loss. Chin Med J (Engl) 2018;131:307-15.

16. Lee JY, Lee YW, Chang SO, et al. Vestibular function analysis of sudden sensorineural hearing loss with dizziness. J Vestib Res 2020;30:203-12.

17. Nogueira-Neto FB, Gallardo FP, Suzuki FA, et al. Prognostic and Evolutive Factors of Tinnitus Triggered by Sudden Sensorineural Hearing Loss. Otol Neurotol 2016;37:627-33.

18. Singh A, Kumar Irugu DV. Sudden sensorineural hearing loss - A contemporary review of management issues. J Otol 2020;15:67-73. 
19. Kleyn AD. Sudden complete or partial loss of function of the octavus-system in apparently normal persons.Acta Otolaryngol 1944;32:407-29.

20. Kostal M, Drsata J, Bláha M, et al. Rheopheresis in treatment of idiopathic sensorineural sudden hearing loss. J Otolaryngol Head Neck Surg 2017;46:50.

21. Schuknecht HF, Donovan ED. The pathology of idiopathic sudden sensorineural hearing loss. Arch Otorhinolaryngol 1986;243:1-15.

22. Chen X, Fu YY, Zhang TY. Role of viral infection in sudden hearing loss. J Int Med Res 2019;47:2865-72.

23. Fukuda A, Morita S, Nakamaru Y, et al. Anti-mumps IgM antibody positive rate with sudden sensorineural hearing loss using second-generation enzyme immunoassay: A retrospective, multi-institutional investigation in Hokkaido, Japan. Auris Nasus Larynx 2018;45:911-5.

24. Esaki S, Goshima F, Kimura H, et al. Auditory and

Cite this article as: Shao M, Xiong G, Xiang G, Xu S, Zheng Y, Zhang L. Sudden deafness as an initial presentation of varicella: case report and literature review. Ann Palliat Med 2021;10(5):58915896. doi: 10.21037/apm-21-785 vestibular defects induced by experimental labyrinthitis following herpes simplex virus in mice. Acta Otolaryngol 2011;131:684-91.

25. Psillas G, Arnaoutoglou M, Gatsios T, et al. Autoimmune recurrent facial palsy and bilateral sudden sensorineural hearing loss following Ramsay Hunt-like syndrome. Auris Nasus Larynx 2012;39:229-32.

26. Rabinstein A, Jerry J, Saraf-Lavi E, et al. Sudden sensorineural hearing loss associated with herpes simplex virus type 1 infection. Neurology 2001;56:571-2.

27. Cashman KA, Wilkinson ER, Zeng X, et al. ImmuneMediated Systemic Vasculitis as the Proposed Cause of Sudden-Onset Sensorineural Hearing Loss following Lassa Virus Exposure in Cynomolgus Macaques. mBio 2018;9:e01896-18.

28. Bhandari R, Steinman GS. Sudden deafness in chickenpox: a case report. Ann Neurol 1983;13:347. 